\title{
EIGHTH DISCUSSION SESSION
}

(Monday morning; 11 September, 1972)

(Following Sections $1-3$ of review paper by Plavec)

\section{Chairman: T. J. HERCZEG}

Herczeg: Now the discussion is opened for the first part of this review paper: first, Dr. Smak.

Smak: I would like to make several comments and ask a few questions. Possible loss of mass and momentum from the system actually affects rather little the final properties of the mass-losing component, these being determined primarily by the properties of its core. The effect is more important, of course, for the dimensions of the orbit, mass-ratio, etc. and - most of all - with regard to the properties of the masscollecting component, particularly in the rapid phase. We really badly need a good description of what is going on around this star before trying to answer the question of how much mass and how much momentum should leave the system at that phase.

Secondly, I want to mention the case of very-low-mass secondaries, for which masses of the order of, or even less than, $0.2 M_{\odot}$ have been determined (examples being AS Eri and DN Ori). These masses are definitely too small, as compared with the theoretically obtained data, and it should be pointed out that the theoretical limit is rather well determined by the maximum possible mass of the helium core. It is my feeling that in some cases at least we should be aware that the accuracy of these observed masses may be too low for us to claim any significant discrepancy here.

Finally, I wish to discuss the problem of undersize subgiants. These appear to be quite common in Algol-type systems and statistically, as far as their physical properties are concerned, do not differ from the contact subgiants. Circumstellar matter is known to be present in systems which contain undersize subgiants as well. It may even be surprising to note that the emission lines originating in the disk are observed more often in those systems than in the semi-detached ones.

The most convincing and self-consistent interpretation of undersize subgiants seems to be that given recently by Paczyński (1971). It assumes that at an earlier epoch, when the subgiant was in contact with its Roche lobe and was capable of losing its mass, a large amount of mass and, particularly, of the angular momentum was stored in the disk surrounding the primary component. After this phase ended, i.e. when the secondary subgiant restored its thermal equilibrium, we had a semi-detached Algoltype system. During the subsequent slow phase of evolution, however, the angular momentum from the disk has been returned to the orbital momentum leading to the increase of the orbital radius and to the expansion of the Roche lobes. It must be noted that this explanation accounts for nearly all observed facts.

A few important conclusions can be drawn from the Paczyński's hypothesis concerning the properties of disks in such systems. First, if we accept that the amount of 
angular momentum stored temporarily in the disk was so large as later to change the orbital dimensions in an observable way, then it means also that the amount of mass stored in the disk was also large. Crude, order-of-magnitude estimates give that it must have been larger than about $1 \%$ of the mass of the subgiant. Second, since the disks are still observed in many such systems, it appears that the time-scale of disruption of such disks is rather long, definitely ionger than the time-scale of processes responsible for the exchange of angular momentum.

I must admit, though, that there is still the possibility that the existence of detached subgiants is a spurious effect and, I understand, Dr. Hall may wish to comment on that.

Biermann: Dr. Thomas in Munich and I are doing calculations very similar to those Plavec has mentioned with the two parameters $f$ and $g$. We had a very simple picture for the angular momentum and mass exchange. We argued about the potential difference that gives energy to the mass flow in the following way: in the fast phase of mass exchange, all the volume around the mass-receiving star is filled with turbulent matter, because the energy gained by the matter flowing down the potential well is very high and not easily dissipated. If this is true, then you can easily set up a differential equation for the parameter $f$ by saying that any matter that is travelling down the potential well gained energy that is dissipated by sending matter out of the system over the potential difference $L_{1}$ to $L_{2}$. We encountered the same problem as you did: that the parameter $g$, which is essentially the effectiveness of loss of angular momentum, is guiding the whole thing. It can very easily happen that the mass-receiving star fills its lobe. We began with the system $\mathrm{BD} 16^{\circ} 516$ which seems to show very strong evidence of high mass loss from the system, because it contains a white dwarf and a low-mass main-sequence star, and yet it seems to be a relatively young system. We have not yet been able to reproduce that kind of a system.

Bath: I was just wondering how dependent do you think the mass-transfer process is on the way in which you treat the envelope structure of the star, and particularly on the specific boundary condition that is imposed to simulate mass loss. It seems to me that the way in which the latter is formulated completely determines the detailed results you have been examining. This is particularly the case for stars with ionization zones and associated convective envelopes, which, according to certain treatments seem to be dynamically unstable to mass transfer. Could you comment on this point?

Plavec: I have treated only case A in this part of my paper. This case of mass transfer involves relatively massive main-sequence stars, which have radiative envelopes. I agree that mass loss from stars with convective envelopes is very high.

Bath: Does this model move during mass transfer into a region of the $\mathrm{H}-\mathrm{R}$ diagram where it has a convective envelope?

Plavec: Yes.

Bath: Isn't it possible that this might affect the later evolution of the system?

Plavec: The model has a convective envelope only at the later stages of slow mass loss. Even convective mass loss is slow then, because the radius of the Roche lobe is already increasing. 
Hall: Dr. Plavec said that in deducing the original mass of the initially more massive component, he found the need to assume considerable mass loss from the system. But couldn't you just as well assume that a significant fraction of the angular momentum coming over in the stream goes into angular momentum of rotation, stored in the outer layers of the hotter star? This seems to me just as reasonable a way out of the problem, because we do know that at least some angular momentum does go into rotation. Furthermore, although rotational angular momentum can be redistributed rather quickly throughout the hotter star, downwards and upwards, we know it may take much longer for rotational angular momentum to be removed from the star, if it ever is. The question is simply how large a fraction of the angular momentum is stored semi-permanently in rotation - whether the fraction is negligible or whether it is large enough to get you out of the difficulty.

Plavec: Prendergast and Burbidge (1968) published a short article telling us that a very good program had been built for computing angular-momentum transfer and radiative transfer in a disk. Because of viscosity the part of the disk closest to the star eventually slows down; the outer part, on the other hand, is accelerating and some mass is lost from the system. This seems to be very significant progress, but, unfortunately, the program has never been published. Everyone is waiting for it and hoping it will be published soon!

Popper: A couple of additional observations on Kopal's concern about not finding the giant successors of main-sequence systems with equal detached components: In the first place, there is not enough room in most detached systems for the components to evolve to the giant stage. There are systems with nearly equal components such as ZZ Boo, EI Cep, and DM Vir in which the components are evolved well through the main-sequence band. And finally there is SZ Cen, a pair of A5 giants, with masses of early A main-sequence stars, that almost certainly lie outside the main-sequence band of core hydrogen burning. It seems remarkable to me that even one system would be found with masses so nearly identical that the components still have nearly equal radii at this stage of evolution.

\section{References}

Paczyński, B.: 1971, Ann. Rev. Astr. Astrophys. 9, 183.

Prendergast, K. H. and Burbidge, G. R.: 1968, Astrophys. J. Letters 151, L83. 